\title{
A Quadratic Equilibrium Entropy Based Virtual Machine Load Balance Evaluation Algorithm
}

\author{
Chao Wang ${ }^{1,2, a}$, Zhou-Yi ZHOU ${ }^{1, d}$, Xue-Gang Mao ${ }^{3, c}$ and Si-Ming Lin ${ }^{1, b}$ \\ ${ }^{1}$ Institute of Computing Technology, Chinese Academy of Sciences, Beijing, China \\ ${ }^{2}$ Zhengzhou Information Science and Technology Institute,ZhengZhou,China \\ ${ }^{3}$ Zhengzhou science and Technology Bureau,ZhengZhou,China \\ awangchao@software.ict.ac.cn, ${ }^{b}$ linsiming@ict.ac.cn, ${ }^{c}$ mxg0371@126.com, ${ }^{d}$ yizhouzhou@ict.ac.cn
}

Keywords: Virtual Machine, Load Balance, Quadratic Entropy.

\begin{abstract}
Aiming at virtual machine load balance evaluation, this paper proposes an evaluation algorithm based on quadratic equilibrium entropy. Firstly, we propose the computational methods for linear equilibrium and quadratic equilibrium entropy. Secondly, we analyze the physical meanings of linear equilibrium and quadratic equilibrium, and analyze their application method in virtual machine load balance. Finally, we prove our scheme by giving experimental results.
\end{abstract}

\section{Introduction}

The load balance equilibrium of virtual machine is one of the most important characters to evaluate the effectiveness of virtual machine scheduling. Existing methods ${ }^{[1][2]}$ usually use load balance degree average and load balance quantity average to measure equilibrium. The results computed this way are static so as not able to reflect variance nature of load balance. We can't compute stability or convergent time based on above results; neither can we deduce the load balance degree of devices with different computational powers. In information theory, entropy is a mathematical tool ${ }^{[3]}$ to evaluate average uncertainty, and has been used in the field of computer science $^{[4][5]}$. [6][7] have proposed concept of parallel computational entropy, and evaluate the reasonability of load balance degree based on the relationship between parallel computational entropy and maximum total computational load on the node. On the other hand, method proposed by [8][9] is mainly used in assistant decision make for resource migration , and has prerequisite that all nodes involved should have equivalent computational power so as not to take the existence of computational power difference and node priority difference.

Ideal load balance algorithm should maintain load equilibrium during its running cycle, while information entropy is the tool to measure the average equilibrium uncertainty. In order to evaluate the load balance effect of virtual machine, this paper proposes theory of linear equilibrium entropy and quadratic equilibrium entropy.

\section{Information Entropy}

2.1 Definition

Information Entropy is the tool to evaluate average uncertainty of event. Its definition is as follows, Let $X$ be discrete random information source, its symbol set be $A: a_{i}(i=12, \ldots, q), q$ is size of symbol set, the probability of event $a_{i}$ is $P\left(a_{i}\right)$, its probablity space [X,p $\left.(x)\right]$ is defined as:

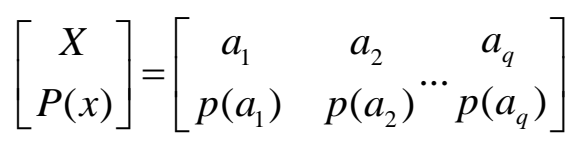

The discrete random information source's information entropy is: 


$$
H(X)=-\sum_{i=1}^{q} p\left(a_{i}\right) \log p\left(a_{i}\right)
$$

Where , $p\left(a_{i}\right) \geq 0(i=1,2, \ldots, q)$ and $\sum_{i=1}^{q} p\left(a_{i}\right)=1$.

\subsection{Weighted Entropy}

The information entropy defined by Shannon is only a function of probability and has not taken human factors into consideration. But in real environment, although the stochastic event happened with certain probability, but different event has different value and effect to people, and is of different importance. It is hard to ignore human factors. In order to take subject value and subject meanings into information measurement, the concept of weighted entropy is introduced.

Let information resource

$$
\left[\begin{array}{c}
X \\
P(x)
\end{array}\right]=\left[\begin{array}{ccc}
a_{1} & a_{2} & a_{q} \\
P\left(a_{1}\right) & P\left(a_{2}\right) & P\left(a_{q}\right)
\end{array}\right]
$$

Where $p\left(a_{i}\right) \geq 0(i=1,2, \ldots, q)$ and $\sum_{i=1}^{q} p\left(a_{i}\right)=1$

And, assign a nonnegative real $w_{i} \geq 0(i=1,2, \ldots, q)$ to event $a_{i}$, the set of real is called weight of event. Let weight of information source's distribution $\left[X, w_{i}\right]$ be

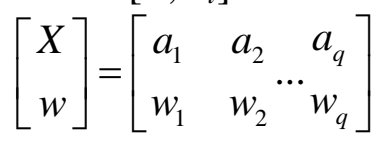

Then information resource $X$ 's weighted entropy is

$$
H_{w}(X)=-\sum_{i=1}^{q} w_{i} p\left(a_{i}\right) \log p\left(a_{i}\right)
$$

\section{Linear Equilibrium Entropy and Quadratic Equilibrium Entropy}

\subsection{Linear Equilibrium Entropy}

Definition (Linear Equilibrium Entropy): Use variable $X$ to represent current information usage of virtual machine.

$$
X=\left\{x_{1}, x_{2}, \ldots, x_{n}\right\}, n \geq 2 \text {, let } p\left(x_{i}\right) \text { be resource usage rate of virtual machine } x_{i} \text {, use variable } w_{i}
$$
to represent virtual machine $x_{i}$ 's computational power factor. If variable $X$ 's probability space $\left[X, p\left(X=x_{i}\right)\right]$ is,

$$
\left[\begin{array}{c}
X \\
P(x)
\end{array}\right]=\left[\begin{array}{ccc}
x_{1} & x_{2} & x_{n} \\
p\left(x_{1}\right) & p\left(x_{2}\right) & p\left(x_{n}\right)
\end{array}\right] \text {, where } p\left(x_{i}\right) \geq 0(i=1,2, \ldots, n) \text {, and } \sum_{i=1}^{n} p\left(x_{i}\right)=1
$$

$w_{i}$ 's distribution is:

$$
\left[\begin{array}{c}
X \\
w
\end{array}\right]=\left[\begin{array}{cccc}
x_{1} & x_{2} & & x_{n} \\
w_{1} & w_{2} & & w_{n}
\end{array}\right] \text {, where } w_{i}>0(i=1,2, \ldots, n) \text {, and } \sum_{1}^{n} w_{i}=1
$$

Then $X$ 's linear equilibrium entropy is :

$$
H(X)=-\sum_{i=1}^{n} w_{i} p\left(x_{i}\right) \log p\left(x_{i}\right)
$$

According to definition of information entropy, the meaning of equation 1 is: the average uncertain measurement of virtual machines in cloud system. Obviously, the greater entropy value, more balanced the load between virtual machines can be, the lesser entropy value, more unbalanced the load between virtual machines can be. Result achieved here is a quantitative character, and linear equilibrium entropy is a relative value which has nothing to do with absolute number of load. Results 
achieved by equation 1 reflect the balance of virtual machine load in one moment. If we want to evaluate whole system's load balance level, we should evaluate the equilibrium entropy of who system's running cycle.

3.2 Quadratic Equilibrium Entropy

Definition (Quadratic Equilibrium Entropy): use variable $Y$ to represent virtual machine load balance measurement on one moment, $H_{Y}(X), Y=\{1,2, \cdots, m\}, m \geq 2$, let $p(y)$ be percentage of virtual machine load balance measurement vs. overall measurement on time $y$,

$$
p(y)=\frac{H_{y}(X)}{\sum_{j=1}^{m} H_{j}(X)}
$$

Then the quadratic equilibrium entropy is:

$$
H_{Y}(X)=-\sum_{i=1}^{m} p(y) \log p(y)
$$

Quadratic equilibrium entropy reflects the stability of virtual machine load balance degree during the system life cycle. This character computes the load balance vibration volume during the virtual machine join in, virtual machine withdraw and algorithm schedule.

\section{Algorithm Verifcation}

We load 10 virtual machines in cloud system, and install load sampling engine in each of them to record the load conditions during a period of time. 20 time stamps are used to sample the system load as shown on table 1.

\begin{tabular}{|c|c|c|c|c|c|c|c|c|c|c|}
\hline $\begin{array}{l}\text { Virtual machines } \\
\text { Num of samplese }\end{array}$ & 1 & 2 & 3 & 4 & 5 & 6 & 7 & 8 & 9 & 10 \\
\hline 1 & 33 & 45 & 29 & 30 & 39 & 44 & 37 & 26 & 33 & 42 \\
\hline 2 & 33 & 35 & 32 & 33 & 34 & 36 & 36 & 32 & 33 & 34 \\
\hline 3 & 24 & 37 & 23 & 17 & 34 & 23 & 37 & 29 & 31 & 34 \\
\hline 4 & 23 & 39 & 24 & 19 & 34 & 33 & 44 & 19 & 34 & 34 \\
\hline 5 & 70 & 65 & 65 & 75 & 80 & 67 & 72 & 73 & 76 & 65 \\
\hline 6 & 40 & 45 & 33 & 23 & 19 & 45 & 35 & 34 & 19 & 29 \\
\hline 7 & 38 & 45 & 38 & 25 & 22 & 23 & 32 & 38 & 22 & 20 \\
\hline 8 & 33 & 40 & 46 & 29 & 34 & 65 & 29 & 23 & 34 & 32 \\
\hline 9 & 38 & 38 & 44 & 44 & 36 & 33 & 27 & 33 & 36 & 45 \\
\hline 10 & 36 & 33 & 41 & 37 & 46 & 27 & 34 & 44 & 32 & 23 \\
\hline 11 & 25 & 34 & 35 & 37 & 43 & 29 & 39 & 45 & 31 & 65 \\
\hline 12 & 27 & 30 & 32 & 31 & 29 & 39 & 33 & 45 & 24 & 33 \\
\hline 13 & 29 & 36 & 29 & 99 & 88 & 45 & 29 & 40 & 23 & 36 \\
\hline 14 & 19 & 29 & 27 & 23 & 26 & 33 & 20 & 38 & 38 & 23 \\
\hline 15 & 40 & 28 & 34 & 27 & 36 & 35 & 45 & 19 & 36 & 46 \\
\hline 16 & 34 & 26 & 39 & 44 & 23 & 41 & 44 & 15 & 46 & 44 \\
\hline 17 & 38 & 26 & 33 & 60 & 31 & 37 & 60 & 23 & 43 & 41 \\
\hline 18 & 23 & 33 & 29 & 29 & 19 & 38 & 29 & 25 & 29 & 35 \\
\hline 19 & 12 & 88 & 67 & 56 & 66 & 62 & 54 & 29 & 36 & 21 \\
\hline 20 & 32 & 36 & 45 & 36 & 44 & 40 & 36 & 44 & 26 & 29 \\
\hline
\end{tabular}

Table 1 Sampling of Virtual Machine Load

According to data gathered in table, let the computational power factor be 1 , compute the linear equilibrium result according to equation 1 , in order to amplify the result, we multiply the result by 10000 as table 2 , 
Table 2 Result of Linear Equilibrium Entropy

\begin{tabular}{|l|c|c|c|c|c|c|c|c|c|c|}
\hline \multicolumn{1}{|c|}{ Virtual Machine } & 1 & 2 & 3 & 4 & 5 & 6 & 7 & 8 & 9 & 10 \\
\hline $\begin{array}{l}\text { Linear } \\
\text { Equilibrium } \\
\text { Entropy }\end{array}$ & 9886 & 9944 & 9816 & 9671 & 9969 & 9613 & 9728 & 9784 & 9874 & 9786 \\
\hline Virtual Mahince & 11 & 12 & 13 & 14 & 15 & 16 & 17 & 18 & 19 & 20 \\
\hline $\begin{array}{c}\text { Linear Equilibrium } \\
\text { Entropy }\end{array}$ & 9808 & 9764 & 9436 & 9641 & 9753 & 9783 & 9246 & 9851 & 9782 & 9805 \\
\hline
\end{tabular}

From table 2, linear equilibrium entropy is related only to balance of virtual machines, and has nothing to do with absolute number of virtual machines. For example in 5th sample, the absolute number of virtual machines is far larger than other samples while its linear equilibrium entropy has not been affected. The result of linear equilibrium entropy directly reflects the load balance degree of virtual machines. If load has obvious unbalance, the linear equilibrium will be significantly lower, take the 17th sample for example.

In real running system, the virtual machines have significant computational power differences. We can adjust the power factor wi to refine linear equilibrium entropy. Under this experiment environment, virtual machines 5 and 6 have significant higher computational power. We get wi's distribution according to inverse relationship between wi and computational power of virtual machine.

Table 3 Distribution of Computational Power Factor $w_{i}$

\begin{tabular}{|c|c|c|c|c|c|c|c|c|c|c|}
\hline Virtual Machine & 1 & 2 & 3 & 4 & 5 & 6 & 7 & 8 & 9 & 10 \\
\hline Computational Power Factor & 1 & 1 & 1 & 1 & 0.5 & 0.6 & 1 & 1 & 1 & 1 \\
\hline
\end{tabular}

We recompute linear equilibrium entropy as table 4.

Table 4 Linear Equilibrium Entropy $(* 10000)$

\begin{tabular}{|c|c|c|c|c|c|c|c|c|c|c|}
\hline Sampling Number & 1 & 2 & 3 & 4 & 5 & 6 & 7 & 8 & 9 & 10 \\
\hline $\begin{array}{c}\text { Linear Equilibrim } \\
\text { Entropy }\end{array}$ & 9926 & 9972 & 9891 & 9834 & 9954 & 9775 & 9876 & 9920 & 9948 & 9919 \\
\hline Sampling Number & 11 & 12 & 13 & 14 & 15 & 16 & 17 & 18 & 19 & 20 \\
\hline $\begin{array}{c}\text { Linear Equilibrium } \\
\text { Entropy }\end{array}$ & 9847 & 9945 & 9672 & 9869 & 9881 & 9806 & 9752 & 9958 & 9282 & 9945 \\
\hline
\end{tabular}

According to linear equillibrium entropy result of table 4, we compute qudratic equilibrium entropy follows quadratic equilibrium equation, as table 5.

Table 5 Result of Quadratic Equilibrium Entropy

\begin{tabular}{|c|c|c|c|c|c|}
\hline Number of Times & 1 & 2 & 3 & 4 & 5 \\
\hline Quadratic Equilibrium Entropy & & 0.999996166 & 0.999994897 & 0.99999069 & 0.999992397 \\
\hline Number of Times & 6 & 7 & 8 & 9 & 10 \\
\hline Ruadratitc Equilibrium Entropy & 0.999986464 & 0.999989235 & 0.999990936 & 0.999991698 & 0.999992799 \\
\hline Number of Times & 11 & 12 & 13 & 14 & 15 \\
\hline Quadartic Equilibrium Entropy & 0.999993181 & 0.9999936 & 0.999986821 & 0.999988081 & 0.999989159 \\
\hline Number of Times & 16 & 17 & 18 & 19 & 20 \\
\hline Quadratic Equilibrium Entropy & 0.999989442 & 0.999988688 & 0.999988809 & 0.999958522 & 0.99996045 \\
\hline
\end{tabular}

To amplify the difference, we multiply the difference between quadratic equilibrium entropy and 1 , and achieve result in table 6.

Table 6 Amplified Difference of Quadratic Equilibrium Entropy and 1

\begin{tabular}{|c|c|c|c|c|c|c|c|c|c|c|}
\hline $\begin{array}{c}\text { Number of } \\
\text { Times }\end{array}$ & 1 & 2 & 3 & 4 & 5 & 6 & 7 & 8 & 9 & 10 \\
\hline $\begin{array}{c}\text { Quadratic } \\
\text { Equilibrium } \\
\text { Entropy }\end{array}$ & & 3.834 & 5.103 & 9.31 & 7.603 & 13.536 & 10.765 & 9.064 & 8.302 & 7.201 \\
\hline $\begin{array}{c}\text { Number of } \\
\text { Times }\end{array}$ & 11 & 12 & 13 & 14 & 15 & 16 & 17 & 18 & 19 & 20 \\
\hline $\begin{array}{c}\text { Quadratic } \\
\text { Equilibrium } \\
\text { Entropy }\end{array}$ & 6.819 & 6.4 & 13.179 & 11.918 & 10.841 & 10.558 & 11.312 & 11.191 & 41.478 & 39.55 \\
\hline
\end{tabular}


Quadratic equilibrium entropy reflects stability of load balance. We can see from the result that there has been serious unbalance on 19th sampling point, quadratic equilibrium entropy has mutational change.

\section{Summary}

This paper proposes a load balance evaluation method based on entropy. This method uses linear equilibrium entropy to evaluate load balance degree and uses quadratic equilibrium entropy to evaluate load balance stability. With both linear and quadratic equilibrium entropy, we can evaluate the quality of different load balance algorithms. The evaluation algorithm proposed in this paper can be applied not only to the field of virtual machine load balance, but also to the fields like network load balance.

\section{Acknowledgement}

This work is supported by the National Natural Science Foundation of China (61202058).

\section{References}

[1]Liu ShiHai. Study on Xen migration mechanism and load balancing strategy.ShanDong University.2012.

[2]Zhang HaiZhou. Research on Cloud Computing Resource Schedueling algrorthm Based on The Urilization and Load Balancing .HarEBin Institute of Technology.2013.

[3]FU Z Y. Information Theory-Fundamental Theory and Application[M]. Beijing: Press of Electronics Industry,2007 (in Chinese).

[4]Wangchao,Chen xingyuan. An Approach for Securty Analysis to Access ControI Policy Based On Entropy-Weigh[J].Acta Electronica Sinica,2013,41(1):47-51.

[5] Wang Chao,Chen XingYuan,Xiong HouRen,Zeng Guang. Quantitative analysis method to access control model based on securty entropy. Computer Engineering and design,2012,33(8):2911-2915.

[6]SUN Hongyuan, XIE WeiXin, YANG Xun, LU KeZhong.A Load balancing algorithm based on parallel comouting entropy in HPC. JOURNAL of SHENZHEN UNIVERSITY SCIENCE AND ENGINEERING,2007,24(1):64-68.

[7]Lu KeZhong,Sun HongYuan. Parallel computing entropy in homogeneous cluster.Journal of ShenZhen University Science and Engineering,2009,26(9):61-64.

[8]Gong Jian, Lu Sheng,Rui Su-Ying. Session-oriented Fast Load Balancing Algorithm. Mini-Micro Systems, 2005, 26(10): 1693-1698.

[9]Zuo LiYun, Cao ZhiBo, Dong ShouBin. Virtual resource evaluation model based on entropy optimized and dynamic weighted in cloud computing. Ruan Jian Xue Bao/Journal of Software, 2013, 24(8): 1937-1946. 